$81 \mid 2012$

Pouvoirs du mythe dans les littératures francophones du Maghreb et du Machrek

\title{
Corps mythiques de Désert
}

Khedidja Khelladi

\section{OpenEdition}

Journals

Édition électronique

URL : http://journals.openedition.org/recherchestravaux/559

DOI : 10.4000/recherchestravaux.559

ISSN : 1969-6434

Éditeur

UGA Éditions/Université Grenoble Alpes

Édition imprimée

Date de publication : 30 décembre 2012

Pagination : 153-168

ISBN : 978-2-84310-238-7

ISSN : 0151-1874

Référence électronique

Khedidja Khelladi, « Corps mythiques de Désert », Recherches \& Travaux [En ligne], 81 | 2012, mis en

ligne le 30 juin 2014, consulté le 08 septembre 2020. URL : http://journals.openedition.org/

recherchestravaux/559; DOI : https://doi.org/10.4000/recherchestravaux.559 


\section{Corps mythiques de Désert}

\section{Premiers pas et cris ultimes}

Désert ${ }^{1}$ : substantif sans déterminant, sujet et prédicat, notion abstraite, lecture ouverte comme la signature de Lalla, l'héroïne du roman de Le Clézio. Qui y trouvera lieu à implication? Le texte assure et rassure, assumant au discours indirect libre les pensées de Lalla : «Ici, il finit toujours par venir quelqu'un [...]» (p. 423). Pour Lalla, sortie à peine du dur travail qui a fait d'elle une mère solitaire et solidaire, obstinément confiante dans la patience du temps, "quelqu'un" viendra, passeur idéal, tout à la fois attendu et inattendu, surgi de nulle part, par hasard et par nécessité.

Et si, le suivant, nous faisions notre entrée dans le désert par ce trop-plein de vie? Leur «ici» devenant nôtre, combien d'ailleurs nous seront-ils offerts? La petite fille courant sur les dunes, jouant avec les oiseaux de mer, qui peut dire s'il s'agit de Lalla elle-même ou d'une de ses épures passées ou à venir? Le promeneur du désert dira l'événement et son récit maintiendra le fil de la mémoire, s'inscrira aux côtés de ceux de Amma ou de Naman le pêcheur, du muet Hartani ou de Ma el Aïnine lui-même, ou de ces voyageurs guerriers du désert ancrant leur récit dans le hors temps des victoires passées, là où la défaite n'en restera pas une. Le recueillant auprès de Lalla, ce promeneur aura la liberté, comme Amma, de ne jamais en donner la même version :

Chaque fois qu'Amma raconte l'histoire d'Al Azraq, elle ajoute un détail nouveau, une phrase nouvelle, ou bien elle change quelque chose, comme si elle ne voulait pas que l'histoire fût jamais achevée (p. I20).

I. J. M. G. Le Clézio, Désert, Gallimard, coll. «Folio», I980; Jemia et J. M. G. Le Clézio, Gens des nuages, Gallimard, coll. "Folio", 1997. Toutes les références, entre parenthèses à la suite des citations, renverront à ces deux éditions. 
C'est la règle entre tout récitant et son auditoire. Cependant, le récitant ne pourra pas badiner avec une seule chose, ce «ils", entrée anaphorique de Désert. Ancêtres ou descendants de $\mathrm{Hawa}^{2}$, fille de Hawa, la première ou la deuxième, ou celle du temps insaisissable d'autrefois, qu'importe : son récit mimé, vécu, trouvera nécessairement une piste où s'ancrer. Le voyageur nomade dispose de tout cela dont la mémoire a besoin : l'émotion est grande devant ce tout premier cri, le lieu est déjà entré dans la cosmogonie, liant et le ciel et la terre et la mer au pied du vieux figuier, et le temps, aube naissante, moment du fijar (p. 2I) appelant à la première prière, en a béni d'autres. Ce "quelqu'un» finissant par trouver la piste menant à Lalla est investi dans le texte du rôle de passeur grâce à la coordination "et » qui insiste sur la dimension protectrice du vieux figuier atavique : «Ici, il finit toujours par venir quelqu'un, et l'ombre du figuier est bien douce et fraîche» (p. 423). Pour dire les liens mythiques entre les hommes, la nature et l'écriture, le corps du texte est composé de deux récits alternés, où la généalogie, les liens du sang maintiennent des échanges et des concomitances que la conception rationaliste du temps aurait refusés.

On les emportait à l'aube, sans cérémonie, emmaillotés dans de vieilles toiles, et on les enterrait dans un simple trou creusé à la hâte, sur lequel on posait ensuite quelques pierres pour que les chiens sauvages ne les déterrent pas (p. 358).

\section{Description-narration-récitation}

Le texte de Le Clézio relève du mythe en ce sens où le langage s'y fait préexistence aux faits. Le réalisme des dures conditions connues de toute vie dans cet espace se transforme, par la présence «diffuse» et «irradiante» de signes dont la lecture participe d'un décryptage-création nécessaire pour comprendre comment ils «s'interprètent» leurs sens. Le mythe, en racontant le désert, non seulement en donne une construction narrative symbolique, mais en révèle un concentré fécond derrière son apparente aridité, pour celui qui voudra bien poser un pied, un regard sur la voie lente du nomade qui avance assuré alors qu'on le croit errer.

Sur ce modèle, le texte est construit de deux récits, se rapportant l'un à la grande Histoire collective, datée, nommément localisée, l'autre à la petite histoire, mi-roman, mi-épopée moderne d'une jeune fille. La chronique se rapporte aux luttes de quelques tribus du désert opposées à l'établissement du Protectorat français sur le Maroc, entre I909 et I9I2 - et la marge blanche

\footnotetext{
2. Hawa correspond à Ève.
} 
laissée à la gauche du texte lui donne d'emblée l'allure d'une geste. Le roman dont on devine grâce à de nombreux indices qu'il se passe à la fin du xx siècle, à Marseille, en France, concerne une de leurs descendantes, dignement rebelle à sa manière. Et le fil conducteur qui permet l'interprétation mythique de ces deux récits les tient solidement arrimés l'un à l'autre.

Des neuf parties composant le texte, la centrale portant expressément sur les conditions de la défaite prochaine ne met pas pour autant fin à l'épopée. Les huit autres alternent, de longueurs d'abord disproportionnées pour se rapprocher progressivement vers un point focal, instaurant une rigoureuse symétrie entre les quatre dernières parties des deux récits : 16 pages et I6 pages, puis II pages et in pages. Les assises de la construction réduisent la distance de départ entre les deux protagonistes qui resteront comme les héros réels de Désert, Nour et Lalla. En premier lieu, toute vision de ce monde est donnée en focalisation interne : leurs points de vue prévalent sur la description (aspects et mise en relation), la narration et la récitation. L'auteur s'occupe en quelque sorte de mettre en ouvre les éléments qui vont faire revivre le désert tel qu'en lui-même il est et demeure. L'hypotypose est dominante, non sélective, infiniment attentive aux grands moments de la marche des tribus comme au moindre mouvement du désert. Ainsi, l'arrivée dans un camp ou sa levée font preuve d'une connaissance fine des comportements des nomades : "Les hommes déchargeaient les chameaux [...] Les femmes allumaient le feu [...]» (p. IO-II); les récits enfin sillonnent le texte échappant au narrateur, distribués en cycles de paroles entre chacun. C'est ainsi que les hommes apprennent en se rendant les honneurs : Amma raconte à Lalla l'histoire de sa mère, Lalla Hawa, Nour informe Ma el Aïnine de sa parenté par la mère avec le grand cheikh Al Azraq, Ma el Aïnine instruit Nour de son éducation auprès du même cheikh et son histoire reprise par les voyageurs fera de lui à son tour un sage; le vieux Naman raconte des histoires enchâssées dignes des Mille et Une Nuits. Rien ne se perd comme le montre l'écho sans voix de la chanson de Lalla Hawa (p. 239) ou la présence obsédante de l'Absent irradiant le texte, rapide comme l'éclair : le signifiant Al Azraq dépasse la couleur bleue du $\mathrm{Ciel}^{3}$ pour être là où il faut, quand il le faut. Il répond ainsi à l'espace-temps sur lequel réfléchissent, chacun de son côté, Nour et Lalla. Pour l'un, "C'était un pays hors du temps, loin de l'histoire des hommes, peut-être un pays où rien ne pouvait apparaître ou mourir...» (p. II) ; pour l'autre, "C'est un temps déjà ancien, et c'est comme s'il n’y avait rien d'écrit, rien de sûr» (p. II ). Pourtant dans ce temps, il n'est pas dit que

3. Le terme connote la rapidité de l'éclair. On dit par exemple : «Essaie de rattraper la bleue ou le bleu» pour parler des coursiers rapides, par référence à la robe d'un noir bleuté de leurs chevaux. 
jamais Nour et Lalla ne se rencontreront. L'auteur se joue avec eux des règles rigides des temps grammaticaux : comment séparer temps du récit et temps du discours quand le corps est pris dans ce qu'il voit, ce qu'il dit, ce qu'il fait et ce dont il a hérité?

Les temps du récit des gens du désert établissent bien la fin de ce monde (plus-que-parfait, imparfait et passé simple), mais le passé composé reconstruit la même période, remettant en question la vanité du jugement des hommes sur la nature. Il rejoint le temps du présent logiquement utilisé pour l'histoire romanesque contemporaine de Lalla : "Ils sont apparus... Ils sont descendus... Ils marchaient sans bruit sur le sable....» (p. 7), exemples étrangement conjugués à ceux de la fin du chapitre : «Nour et son père s'arrêtèrent encore... Puis, ils ont dit la dernière prière...» (p. 32). Ainsi sont ravivées mémoires et rencontres dans cet autre temps : celui des "Maintenant» qui ponctuent les deux récits, particulièrement le premier ("C’est là qu'ils arrivaient maintenant...») (p. I6) ou dans le second constatant la limite de Lalla et la victoire impossible : "Maintenant il y a le haut mur de séparation du parking...» (p. 394). De déictiques spatiaux, ils deviennent chronotopes enregistrant l'histoire des hommes grâce à la reprise des "Alors" qui balisent la narration.

Ainsi ramenés l'un vers l'autre, Nour et Lalla sont au centre de récits de vie et de survie d'hommes affrontant la nature sans la combattre ou se mesurant au progrès en respectant le temps. Dans le cadre de cette poétique pour dire la quête d'un territoire ${ }^{4}$ où Nature et Culture se respectent, la frontière des genres paraît bien secondaire et Le Clézio ne s'en inquiète pas, qui fait d'abord œuvre de poète avec ce que cela suppose de constructions en échos, de correspondances, de corrélations. Les investissements avec lesquels s'amplifie tout avènement permettent aux hommes de l'Histoire de suivre des pistes "presque invisibles» (p. 7). Corps inscrits dans une mémoire de la longue durée du passé glorieux, traversant ce présent houleux mais ayant déjà jeté un fil vers un devenir en train de naitre : c'est que tous les protagonistes historiques ou fictifs sont désormais dépendants du dire d'une épopée du désert qui se mythologise progressivement en Désert. Il est naturel que dans ce monde, le premier filon vers la mythologisation soit la généalogie et ses questions de légitimation.

4. En anthropologie, le territoire est défini comme étant «à la fois objectivement organisé et culturellement inventé»; A. Bougeot, "Territoire», dans Dictionnaire de l'ethnologie et de l'anthropologie, PUF, 1991, p. 704. 


\section{Les-gens-de : l'hagiographie}

Par l'anaphores d'ouverture, Désert s'inscrit dans un de ces moments de récitation par lesquels le lecteur averti des traditions orales se retrouve en fait dans une longue chaîne de héros. Précédant ou succédant (à) Hawa fille de Hawa? La question paraîtra peu à peu secondaire devant la puissance d'un certain type d'héroïsme. Celui-ci ne tient pas d'abord à leur rôle de personnages dans le texte, mais surtout à leur histoire vécue, depuis des temps immémoriaux, d'où leur entrée triomphante dans le mythe en dépit d'un certain type de défaite. Le «ils» de leur surgissement en la matière du texte ne les atomise ni ne préjuge de leur dispersion; c'est un moment de forces égales, opposées, cherchant un équilibre dans un parcours hors du temps, si ce n'est celui du désert, et que de nombreux indices et catalyses permettent de reconstituer, mais non d'appréhender, car ce serait le banaliser. Or ces nomades tirent leur liberté, tout à la fois, de leur légitime chefferie et de leur mise au ban de la société : héroïsmes et marginalités constituent bien deux pôles ouverts à l'épique dans ce qu'il a de transculturel et dans ses possibles investissements conjoncturels, spécifiques. Les indices les plus parlants, comme nommer des personnages sous l'égide desquels toute la diégèse est inscrite, ramènent immanquablement à ces légendes hagiographiques dont des auteurs comme Émile Dermenghem ou Jacques Berque ont très tôt compris la place dans la constitution des codes de fonctionnement des sociétés maghrébines. Le Clézio lui-même est allé en chercher les traces vivantes dans Gens des nuages que l'on ouvre sous le signe de Sidi Abou Madyan et Rumi ${ }^{6}$ (p. 27), sous les aphorismes desquels sont placés les différents paragraphes du texte.

En tant que héros du texte, Nour est le premier nom distingué des autres (p. 9), distinction déjà liée au rôle de son père : «Un seul d'entre eux portait un fusil» et «Nour, le fils de l'homme au fusil» (p. 9). Lalla, dont l'appellation impose le respect, bénéficie à elle seule des chapitres les plus importants. Par ailleurs, en tant qu'acteurs de l'hérö̈sme, ils sont crédités des attributs classiques de ce statut : naissance particulière, exclusif ou exclu : rien d'original en somme par rapport à tout récit (conte, légende), puisque Ma el Aïnine,

5. C'est une pratique commune à toute la littérature orale où la continuité semble établie de fait entre auditoire et récitant. Nous nous permettons de citer des débuts de textes que nous avons recueillis : «Ils eurent de mauvaises années», "Ils répondirent à l’invitation de Jazia».

6. Sidi Abou Madyian : mystique né dans la région de Séville en II26, mort en II97 en Algérie, à Tlemcen dont il est le saint patron. Rumi : poète d'origine grecque byzantine, né en 836 , mort en 896 . 
Nour et Lalla sont des nobles, Hartani un affranchi et Radzic, un gitan et enfant vendu. Pourtant, le même pressentiment les met face à leur destin qu'ils vivront jusqu'au bout. Nour entre presque par effraction dans le monde du fondateur, se réveillant à temps, traversant les remparts, par une "porte étroite" pour suivre mot à mot les paroles du guide suprême : "Nour sentait cela surtout quand il regardait la silhouette fragile du grand cheikh, comme s'il entrait dans le cœur même du vieillard et qu'il entrait dans son silence» (p. 39). Lalla est saisie, troublée par l'appel qu'elle ressent en elle, et interroge celui qu'elle appelle "son secret» : "Elle voit ce qu'il y a dans le regard de l'Homme Bleu» (p. 203) avant de ressentir quiétude et sérénité après son passage. S'ajoutent à ces caractéristiques des attributs physiques distinctifs. Nour est la lumière dont il porte le nom; concentrée chez tous en un regard à la limite du surnaturel, cette lumière éblouit ces êtres extraordinaires qui arrivent à en projeter les rayons sur les autres, qu'elle vienne du ciel, du soleil ou des étoiles et même des artifices de l'homme de la ville. L'initiation qu'ils vont recevoir ne dépend pas du statut ni n'attend le nombre des années. Directement ou par personne interposée, sans contrainte spatio-temporelle, ils se retrouvent : la jeunesse des uns, retenue ou exubérante, guidée par la toute puissance des initiateurs.

"À quoi sert l'épopée? ?", se demande Jean Dérive. La recension de ses premiers éléments, relevant tout à la fois de la logique narrative et des indices axiologiques, permet de classer Désert parmi les textes proches de l'épopée :

Cette fonction du modèle épique de type historico-mythique consistant à faire lire le présent de l'énonciation à la lumière d'un épisode du passé largement mythifié semble bien avérée à différentes époques et en divers milieux culturels.

Le premier indice dans le texte est un lieu dont l'occurrence est facilement notable : Saguia el Hamra ${ }^{8}$. Donné d'abord comme titre au premier chapitre, le toponyme est repris à partir de la page $\mathrm{I}_{4}$ : «Maintenant, ils étaient apparus au-dessus de la vallée de Saguiet el Hamra... » avec sa traduction en page 20 : «la vallée rouge» et les précisions qui suivent en page 25 : «Les routes étaient circulaires, elles conduisaient au point de départ, traçant des cercles de plus en plus étroits autour de Saguiet el Hamra»; "Ils étaient revenus, chargés de munitions, jusqu’à la terre sainte de la Saguiet el Hamra.» Puis page 27, la présentation suprême : «C'était le centre du désert, peut-être, le lieu où tout avait commencé, autrefois, quand les hommes étaient venus pour la première fois.»

7. J. Dérive, L'Épopée, unité et diversité d'un genre, Kartala, 2002, p. I77-I88.

8. Lorsque nous citons Le Clézio, nous gardons l'orthographe adoptée par l'auteur «Saguiet el Hamra»; autrement, nous conservons l'orthographe la plus communément usitée : Saguia-el-Hamra. 
Cette référence et les différents récits mis en abyme y afférents mettent en lumière les sources même de la géographie mythique de Désert : épisodes historiques fondamentaux dans la mise en place des sociétés maghrébines qui repris en charge par la transmission orale ont investi l'imaginaire des populations et constituent un ensemble tout à la fois homogène et comportant de nombreuses variantes. Immense réservoir pour qui veut comprendre la mise en place des codes de fonctionnement de ces sociétés, comme le fait Le Clézio ici. À quoi correspond cette dénomination? Historiquement, Saguia el Hamra a connu deux types d'arrivées plus ou moins massives de lettrés et de savants en arabe et en berbère, peu ou prou liés au mysticisme musulman : aux $\mathrm{XI}^{\mathrm{e}}$ et XII ${ }^{\mathrm{e}}$ siècles, d'abord venant de Bagdad, puis, au xve siècle, pour fuir la Reconquista. Le schéma type relatif à leurs parcours les fait osciller entre pérégrinations et errance projetées dans un premier temps vers l'acquisition d'un territoire pour s'y installer et prêcher la bonne parole auprès des populations. Ensuite, un véritable balisage de l'espace se fait avec la mise en place de centres d'étude religieux, les zaouias.

De par sa situation géographique, à l'extrême Sud-Ouest du Maroc, Saguia el Hamra fut un passage obligé pour ces éternels voyageurs : de l'Andalousie, ils essaimèrent, dans toute l'Ifrikia', laissant de leur passage des traces tangibles, la fondation d'une cité. Le schéma de l'implantation et les qualités nécessaires pour ce faire sont identiques. L'acquisition d'un territoire ne se fait pas toujours aisément, des rejets furent enregistrés, obligeant justement à une incertaine nomadité, terme par lequel sont désignés tout à la fois les déplacements obligatoires et les règles de comportement qui en découlent, comme l'endurance et la frugalité érigées en code, celui de l'ascétisme indispensable à la spiritualité. L'enseignement suit cette pédagogie de l'exemple et s'impose par des dons oratoires certains. Anthropologues, historiens, sociologues reconnaissent à ces mouvements une responsabilité indéniable dans les fonctionnements, les mentalités et l'imaginaire des sociétés maghrébines. La plupart des analyses insistent sur leur rôle intégratif, leurs actions consacrées à l'adoration de Dieu ou encore leurs pratiques : "Le soufisme recherche par l'exercice de la vie contemplative et des pratiques précises un état de pureté morale et spirituelle ${ }^{\mathrm{Io}}$." Si les manuscrits existent pour lire cette histoire, toute une littérature populaire s'est répandue, tournée vers les prouesses, les miracles, les difficultés et offrant, à travers les variantes, la possibilité de connaître la réception de ces mouvements et permettant que le modèle type soit remis en œuvre si le besoin historique s'en fait sentir. Concernant

9. L'Ifrikia représentait l'actuelle Tunisie et une partie du Constantinois algérien; le Maghreb central correspondait à l'Algérie actuelle.

Io. M. Kaddache, L’Algérie médiévale, Alger, Enal, I992, p. I33. 
précisément l'histoire du Maroc, Évariste Lévi-Provençal affirme la place de ces mouvements et leur rôle :

Avant la fin $\mathrm{du} \mathrm{xv}^{\mathrm{e}}$ siècle, les derniers Maures quittent la péninsule ibérique. À ce moment va se former une école vraiment marocaine. Elle a pour elle, tout d'abord, l'appoint considérable d'une grande partie des expulsés du pays voisins. Si elle reste en relations suivies avec les centres intellectuels d'Orient, elle échappe néanmoins à leur tutelle; et dans le pays, elle va rayonner, non seulement de la capitale savante et de son Université mais aussi, et encore plus, des zawiyya disséminées dans le pays. L'écriture de l'histoire restera longtemps tributaire de leurs actions, élevées au niveau du sacrér ${ }^{\text {II }}$.

Les nomades de Désert sont de ceux-là, que les indices soient explicites (ne serait-ce que la mention à Sidi Abdel Kader et à Chibli ${ }^{12}$ ) ou implicites comme les différentes errances; car, rapportés par la tradition écrite des biographes ou repris par les légendes hagiographiques orales, ces mouvements constituent une véritable topique socioculturelle. Une coupe dans celle-ci comme le préconise Gilbert Durand ${ }^{13}$ permettra de rapprocher, en dépit du temps, Ma el Aïnine et Nour de Lalla et du Hartani. Fonctions et attributs se constituent en figures mythiques, ces corps épandus regroupés autour des rôles du guerrier, du berger et du saint, poète en même temps :

Récitant de Désert, toi ce quelqu'un providentiel qui donnera sens au voyage de Lalla en en faisant le récit, tu auras à respecter le génie des uns et des autres puisque tu sais que les errances ne se ressemblent pas.

\section{Il y a errance et errance}

Du début à la fin du $\mathrm{Xx}^{\mathrm{e}}$ siècle, entre Nour et Lalla, cette topique socioculturelle démontre les mécanismes relationnels des différents processus de mythification : les échos mis en place par l'auteur, d'une part, entre les visions du désert et, d'autre part, celles de la ville se répondent par des mouvements de rapprochements et de renversements des investissements sémantiques et actanciels. Conçu comme un ensemble vide, le désert se remplit des regards du groupe autour de Ma el Aïnine et de ceux de Lalla et de son monde; historiquement, il devient espace urbanisé avec les constructions de cités telles

II. É. Lévi-Provençal, Les Historiens des chorfa suivi de La Fondation de Fès [1932], Maisonneuve et Larose, 200I.

I2. Sidi Abdelkader al-Djilani né à Guilan, sur les bords de la mer Caspienne et mort à Bagdad en II66. Chibli, poète né à Bagdad en 86I et mort en 933; voir E. Dermenghem, Le Culte des saints dans l'islam maghrébin, Gallimard [1954], 1982.

13. G. Durand, Introduction à la mythodologie, Albin Michel, Tunis, Cérès, 1996. Voir le concept de topique socioculturelle, p. I57-I84. 
que Smara. Ainsi une dynamique de multiples conflits se met en place, vécus les uns dans une séparation totale comme ceux relevant de désert versus ville ou ville ancienne versus ville moderne ou opposant de façon naturelle, relevant de l'ordre des choses, le désert de Nour et celui de Lalla.

Comme pour tout nomade, la voie du Récitant ne fut pas errance aveugle, perte déboussolée; elle ne fut pas erreur de trajet mais trajectoire, rêvée comme celle de Lalla, envolée folle comme celle du Hartani, itinéraire organisé comme celui de Nour auprès de Ma el Aïnine. Il est difficile pour un sédentaire de saisir ce qu'il y a de construit dans le vide apparemment désaxé du désert, ardu de percevoir l'ordre de ce chaos.

Le surgissement du récit ne s'échappe pas d'une béance, il se dévide en suites ininterrompues, continuums des rencontres entre les hommes, leurs espaces et leurs mots. Les hommes qui les écoutent en reconnaissent les résonances portées par le moindre grain de sable, et la récitation réitérée leur offre la possibilité renouvelée d'y inscrire leur place, et pour cela, le récitant de Désert tient son lecteur-auditeur en un cadre où il tache d'apposer autant de signes possibles. Il est vain de rechercher l'antériorité des uns ou la succession de l'ordre des événements, d'ailleurs les temps utilisés par l'auteur ne le permettraient pas. Les temps passés dans les campements, les mouvements des caravanes ou les jeux (poursuites des enfants ou arrêts auprès du tombeau d'un saint), tout cela a donné autant de stations sensées, signifiantes et orientées. À preuve, ces mouvements d'appels et d'échos, de parallélismes et de renversements entre l'errance de la caravane et celle de Lalla. D'un désert l'autre, il reste ces marqueurs entre la sagesse finissante du vieux fondateur et la révolte toute en innocence d'une de ses descendantes : une terre et la liberté.

Du côté de la tradition, l'histoire marquée par une politique de défense et la traversée du désert est mouvement non de repli mais de retour sur un sol viable et à défendre. L'avancée tout en souffrances, en combats contre les dures conditions de vie ne présente aucun sentiment de tragique. La nature est respectée et suivie comme le permettent les étendues désertiques, la symbiose entre les hommes et le désert se révèle dans la facilité avec laquelle ils entrent sans le mouvement infini du sable : "Ils marchaient sans bruit dans le sable» (p. 7). Leur appartenance à ce monde maintient cette adhésion aux règles de l'environnement, ce respect de ce qui préexistait à l'homme en dépit des dures contraintes d'y survivre : «La faim les rongeait. Ils n'auraient pas pu parler. Ils étaient devenus, depuis si longtemps, muets comme le désert...» (p. 8).

Pourtant, la vie est d'un ordre impeccable, nécessaire : «En tête de la caravane, il y avait les hommes, enveloppés dans leurs manteaux de laine, leurs visages marqués par le voile bleu. Avec eux marchaient deux ou trois dromadaires, puis les chèvres et les moutons harcelés par les jeunes garçons. 
Les femmes fermaient la marche» (p. 7). Des règles de culture s'imposent; en premier lieu le partage entre les hommes, cela va de soi, mais surtout avec les bêtes: "Les hommes et le troupeau fuyaient lentement..." (p. Io); nourrissant les hommes, les bêtes deviennent nourricières de la terre : "Les bêtes aussi étaient mortes, gorge ouverte pour fertiliser les profondeurs de la terre» (p. 13). En deuxième lieu, la connaissance qui permet de lier et le ciel et la terre, de lire le comportement des éléments naturels. Nour la reçoit de son père, déjà singularisé par le port du fusil. Enfin, la culture initiatique dont seul bénéficiera ce "fils d'une chérifa ${ }^{14}$ comme l'établit Ma el Aïnine : «Ainsi, ta mère est de la lignée de Sidi Mohammed, celui qu'on appelait Al Azraq, l'Homme bleu?» (p. 54) La généalogie a refait surface et les itinéraires de Nour et de Ma el Aïnine seront désormais liés à ce sol. Si le premier tient son nom des étoiles, le second est une sorte d'antonomase que les légendes ont extraite du sol même du désert. En effet, en dehors du nom propre du fondateur, l'expression «Ma el Aïnine» est apparue à plusieurs reprises dans le texte pour noter non seulement combien l'eau est précieuse au désert, mais aussi le lien sémantique qui, en arabe, la lie aux yeux et à la source. Dans les citations suivantes l'auteur insiste sur la polysémie du terme "aiun ", singulier «ain» signifiant en arabe tout à la fois la source, la fontaine et l'œil : «L'eau, elle était dans les aiun, les yeux...» (p. I3) «C'était comme les yeux de l'eau au milieu du désert» (p. I7). L'initiation enfin qui attire le regroupement collectif par le respect du nom des tentes dans cette convergence vers celui qui a été élu pour les représenter et le mérite grâce à sa pédagogie de l'exemple, comme lui-même la reçut de Al Azraq son maître : "Mais tu ne m’as pas encore donné ton enseignement», dit Ma el Aïnine. Alors Al Azraq lui avait montré le plat de dattes, l'écuelle de lait caillé et la cruche d'eau : "N'ai-je pas partagé cela avec toi, chaque jour depuis que tu es arrivé ?" " (p. 55).

Le même désir d'enseignement attire dans la solidarité les différents groupes : "Alors, ils sont venus de plus en plus nombreux dans la vallée de la Saguiet el Hamra...» (p. 33 ). Ils se retrouveront unis par le corps dans la transe mystique qui les lie au sol par leurs pieds nus et au ciel par la voix qui devient soupir porté par les allitérations en "h»; la paronomase des chants des femmes, «forge des gorges» (p. 70), renforce le caractère sacré du chant : "Je suis venu ${ }^{15}$ ", et, grâce à ce performatif ${ }^{16}$, l'incantation se fait graduelle du

I4. Une Chérifa est une descendante du Prophète.

I5. Invocation habituelle adressée à tous les saints, ainsi cette chanson pour Sidi Abou Madyan : «Sidi Abou Madyan, je viens vers toi en quémandeur, montre-toi à moi, dans mes rêves, que je guérisse.»

I6. Tout déplacement vers le saint est une action obligatoirement précédée d'une déclaration d'intention qui, non proférée à voix haute, serait nulle et non avenue. 
Divin à ce qu'il peut y avoir de divin en les hommes (p. 58-7I). Cependant, nulle condescendance n'est permise, les trahisons tardives seront rapportées (p. 382), tout comme les remarques admiratives de l'observateur des soldats des chrétiens et les miracles de Ma el Aïnine ramenés comme il convient dans son enseignement à une dimension allégorique : l'aveugle qui suit Nour comme son antithèse lui offrant la chance de partager sa luminosité, une fois béni, "sera un homme qui sait où il va» (p. 372), sans la faculté de voir.

Ces valeurs fondamentales marquées à jamais dans ces stations, en traces, seront revisitées par Lalla, héritière de la même lignée que Nour, héritage maintenu en ces temps modernes sous le sceau du secret. Lalla, désignée par son statut qui exige le respect, arrive au désert dans une situation de manque, la perte de sa mère; élevée par sa tante paternelle désignée également par cette relation, "Amma», elle fait corps avec ce milieu physique où elle évolue et évoluera jusqu’à le quitter et y revenir comme le lui avait annoncé son récitant préféré, le pêcheur Naman : «Toi, tu iras. Tu verras toutes ces villes, et puis tu reviendras ici, comme moi» (p. IO4). On sait que cette prédiction des «hommes-récits», comme les appelle Todorov, sera réalité ${ }^{17}$.

Lalla, dans le long développement (p. 75-22I) consacré à son bonheur, se saisit des quatre éléments. Ces derniers constituent chacun une tête de paragraphe : "Le soleil se lève au-dessus de la terre... Lalla marche lentement" (p. 75). Déjà l'adverbe la relie à la marche des ancêtres (p. 7). Les autres éléments défilent un à un, ouvrant la fuite éperdue de la petite fille : "Il y a un endroit où Lalla aime bien aller, il faut prendre les sentiers qui s'éloignent de la mer et qui vont vers l'est, pour remonter le lit du torrent séché» (p. 94); "La lumière est belle, ici sur la cité», jusqu’à ce que le Hartani lui apprenne à la regarder (p. I26). Enfin, «Lalla aime le feu» (p. I42). Il y a surtout ce vent, tantôt souffle de vie, tantôt poussière de mort, jusqu'à devenir «ce vent de malheur» dont Nour et Lalla savent qu'il est annonciateur de mort, celle de Ma el Aïnine, puis celle de Hartani. Supportant privations et souffrances, toujours en attente de quelque chose dont elle a la certitude qu'il adviendra, Lalla transmet son euphorie à la nature. Dans ce désert qui est le sien, le vivant microscopique advient à la nature sous son regard ou celui de ceux qui constituent son monde, hommes réels, hommes de récits, hommes de l'invisible. Les insectes font partie de ce monde scruté, comme observé à la loupe : guêpes, mouches, bourdons attirent son intérêt, tout comme les plantes si rares. Sa première apparition ne la met-elle pas en scène devant les plantes grasses luisantes, antinomiques avec l'aridité (p. 75) ? Personnifiés, porteurs d'un langage qu'elle s'essaie à faire sien, les oiseaux l'accompagnent de son

17. T. Todorov, Poétique de la prose, Seuil, I971, p. 33. 
enfance à la vie d'adulte. On les retrouve à tous les moments de ce bonheur qui, présenté par l'auteur en focalisation interne, a pour lui les qualités naturelles, évidentes comme le prouvent les occurrences du simple verbe "aimer», suivi de l'explicatif tout aussi simple "parce que» : «Lalla aime bien marcher entre les collines...» (p. 136). Mais elle ne s'approche pas trop près, parce qu'elle a un peu peur tout de même (p. 75). Sa jouissance du monde extérieur est extrême : rien d'impur dans la nature ni dans les corps humains dont toutes fonctions sont valorisées, sueur, urine et les cinq sens aiguisés; elle veut toucher le vivant, le sentir, jusque dans ses viscères (p. 19). Pour autant, l'insouciance n'est pas béatitude. Des interpellations la troublent dont elle recherche les réponses auprès de ses compagnons de route : d'abord lui rapporter les récits des siens, et surtout de sa mère; connaître les ailleurs en traversant les mers par les mots qui savent les dire. La mélancolie est grande et la souffrance terrible : si les mots ne suffisent pas pour les atténuer, il reste ses deux personnalités qui l'amènent vers le dénouement de toutes ses crises. Il y avait depuis toujours l'ombre protectrice de l'ancêtre premier Al Azraq qui, par-delà le temps, maintenait, par sa présence, invisible le lien généalogique avec Nour, qui intervenait afin de lui faire comprendre son statut d'élue. Il permet que les deux enfants en dépit du temps chronologique ressentent la même angoisse devant «le vent du malheur» qui poursuivra Lalla jusque dans la ville.

La célèbre chanson de la mère de Lalla, énonçant tous les possibles états de la nature, sauf la fin de son amour ( $U$ Un jour, oh, un jour le soleil sera obscur... car ce sera le jour où je quitterai mon amour", p. 176) est perçue de façon prémonitoire par Lalla lorsqu' "elle ne sait pas où elle va, à la dérive...»" (p. 204); mais, après avoir entendu qu' "Un jour, oh, un jour le corbeau deviendra blanc» (p. 205), elle fera corps avec la lumière qui l'envahissait.»; "C'est de là que semble venir la lumière du regard, et Lalla comprend que c'est la demeure de l'Homme Bleu» (p. 205). Véritable chanson de toile, elle tisse les liens entre les générations. Il y a surtout le marginal, affranchi, prétendu fou, accusé de magie, le Hartani, qui possède les qualités surhumaines, monstrueuses de par leur rapprochement avec l'animalité. Lui aussi est gratifié de la même surhumanité que Nour, celle-là même que par un simple toucher de main Ma el Aïnine transmettait à un homme privé de la faculté de voir pour retrouver sa route. À eux trois, ils bénéficient de la même puissance de regard : concernant Nour, il est dit que «la lumière de son regard était presque surnaturelle» (p. 9), de l'Homme Bleu, on retient que Lalla sait qu’il «la protège de son regard» (p. 95). C'est qu'en réalité dans le désert de Lalla et du Hartani, la synesthésie est reine de la sensibilité : ils apprennent à devenir autre, animal, rocher, mimer les corps et les nommer 
sans les mots de la parole, trouver un langage propre à leurs idéaux : «et c'est dans la lumière de son regard qu'on entend ce qu'il dit» (p. I32); ou encore : "Ce sont plutôt des images qu'il fait naître dans l'air... mais jamais Lalla n'a rien entendu de plus beau» (p. 133).

Alors son départ vers la ville rêvée comme le mot d'une chanson (Méditerra-né-é-é...) (p. 82-83) donne la mesure de ses désillusions. Si Smara représentait la cité-paradis fondée par le groupe identifié des tribus, sortie du désert pour offrir la vie, la ville du progrès s'établit sur des relations oxymoriques; elle est gouffres et ténèbres, foules solitaires, richesse et mendicité. Les échos établis par l'auteur, s'ils sont poétiques au niveau de l'organisation du texte, insistent sur le malheur. Lalla y passe par des métamorphoses sans jamais s'y perdre : toujours sensible à la marginalité, elle se prend d'amitié pour le jeune gitan, enfant vendu, voleur et mendiant, victime des adultes et à qui d'autres adultes ôteront la vie au nom de la justice. Lalla lui aura prodigué des moments de richesse, jetant l'argent gagné grâce à son image, son reflet, pensant peut-être comme Baudelaire que le miroir, trompeur, fait «toute chose belle». Ou bien se rappelant sans le savoir que les siens avaient perdu la liberté à cause de l'argent de ces villes : "Ce n'était pas les armes qui l'avaient vaincu, mais l'argent» (p. 379). Les parallélismes, tout en inversion, font qu'un de ces mondes est bien le revers de l'autre. Et c'est encore avec la perception de son propre corps que Lalla retrouve sa vraie liberté, dans une danse dont la chorégraphie n'est pas loin de rappeler la transe mystique du groupe de nomades après le dzikr de Ma el Aïnine. (p. 58-70) «La tête tournée à droite, à gauche, à droite, à gauche, et la musique qui était à l'intérieur de leurs corps traversait leur gorge...» (p. 68), est-il précisé de la prière collective et Lalla, dans la ville des esclaves, sous les feux des projecteurs vit le même transport : «Elle danse sur le rythme lent de la musique électrique, et c'est comme si la musique était à l'intérieur de son corps... Hawa danse pieds nus sur le sol lisse...» (p. 355). Tous ces rapprochements irrecevables dans une mesure rationaliste du temps fondent une poétique qui la prépare à recevoir son secret, et elle repart de nouveau. Errances, cette fois, va-et-vient incessants, avant de réaliser la prédiction du pécheur. Peut-être devient-elle elle-même ce prince des oiseaux qu'elle nommait el Haim, l'errant : "Haim : Haim! Viens, je t'en prie!» (p. I7I)

Sous son manteau, la véritable errance, celle que maitrise le nomade, recommence. 


\section{Sous le manteau, le fil du secret}

Tandis que les siens disparaissent, ne laissant aucune trace visible de leur passage, tel le tombeau du cheikh introuvable par Nour, ou les nomades enfouissant leurs excréments par respect pour la nature (dernière page), Lalla revient, ni départ en retraite, ni échec de retour. Le désert est devenu le Lieu Désert, passage des trois monothéismes que Lalla rencontre de façon exceptionnelle, le cheikh musulman Al Azraq, Naman, le pêcheur juif et le photographe chrétien. Passeurs, ils laissent une cohérence de signes hétéroclites qui retrouvent une fonction d'ensemble pour que le lecteur lève le sens qu'ils diffusent, une spiritualité sans dogme. Retenons ceux qui s'imposent, ne serait-ce que par leur occurrence ou leur désignation : tandis que le texte avance, l'ombre du vieux figuier occupe l'espace (p. I05, I07, 2II, 4I5, 416 et trois fois pages 83 et 419) et le vieux manteau se déploie (p. 7, 266, 268, 269, 3IO) : "Son manteau marron est étendu par terre sur le sol caillouteux. Elle accroche la ceinture à la première branche maitresse du figuier...» (p. 42I)

Des termes arabes connotant une référence fondamentale dans la vie du nomade sont en général transcrits en caractères latins dans le texte, ainsi de la plante chiba (p. I30), de la prière de l'aube el fijar (p. 2I), du $d z i k r$ (p. 57), cette répétition litanique des mystiques. Les noms propres sont paradoxalement traduits, mettant en valeur leurs connotations, comme L'Homme Bleu ou l'Eau des Yeux. Cependant, le terme "manteau» dont l'occurrence a été relevée dans le texte et qui est entré dans la langue française sous la forme de l'emprunt culturel «burnous» n'apparait jamais sous cette dénomination. Présenté dès la première page du roman ("C'étaient des silhouettes alourdies, encombrées par les lourds manteaux", p. 7), il retrouve rapidement ses fonctions utilitaires pour la caravane en déplacement ( $\ldots$...ils s'étaient enroulés dans leurs lourds manteaux de laine.", p. I8); il réapparaît blanc symbole de la spiritualité du cheikh, qui impressionne Nour : «...et il vit le blanc manteau du cheikh» (p. 53). Il se retrouve par ailleurs, couvrant Lalla en toutes circonstances dans sa vie chez les esclaves, lui permettant d'assister, anonyme, forme devinée, à la mort de son ami le gitan : «il y a une jeune femme très sombre, immobile comme une ombre, qui regarde de toutes ses forces» (p. 396). Plus qu'un signe de protection, le mot dépasse la fonction de l'objet. Une mise en regard avec d'autres situations laisse penser que le mythe, récit ou écriture, est modulé par une dimension philosophique certaine. Les indices trop importants dans le texte ne peuvent tous figurer dans cette lecture d'ordre général. Cependant, le lecteur est pris dans une réflexion qui dépasse le voyage des nomades et de Lalla. Comme Désert, ces acteurs 
s'inscrivent en dehors du temps tandis que précisément leur vie et leur enseignement jouissent d'une véritable "extase matérielle». Il est justement précisé que Lalla aime les signes : "Mais Lalla n'a pas peur des signes, ni de la solitude...» tout comme Le Hartani d'ailleurs : «Lui non plus n'a pas peur des signes sur les pierres» (p. 95-96). La place que ce manteau tient dans le roman ouvre la lecture du mythe à différentes modulations relevant du sacré religieux et de la philosophie. Sacré et philosophie se complètent ici, fondés sur le respect de la vie, la quête du spirituel et de l'extase à partir de l'amour de toute matière vivante. Un certain sentiment du religieux, du soufisme et du cynisme se rencontre bien sous ce manteau qui traverse le texte. Les différents corps mythiques de Désert ne nous ont-ils pas ramenés à ces noms où les changements tiennent toujours du possible?

Le chant liturgique relatif à toute protection que peut apporter Le Prophète s'intitule précisément El Bourdah («Le Manteau»). Saint François d'Assise ouvrait son manteau aux pauvres et aux oiseaux. À ces schèmes strictement religieux, renforcées par la présence de nombreux saints de l'islam évoqués tout au long du texte, se surimposent d'autres relevant du cynisme comme philosophie. Nous retrouvons, en effet, des ressemblances avec ces descriptions que Bréhier donne de Socrate ${ }^{18}$ " qui, vêtu d'un manteau grossier, parcourait les rues pieds nus», ou de Diogène de Sinope qui privilégie la "tenue ordinaire des hommes du peuple (manteau qu'il replie sur lui-même pendant l'hiver)».

Est-ce étonnant si le retour de Lalla dans la Cité de tôles et de zinc se fait sous le signe de la recréation du monde : voyageuse invétérée, proche des laissés pour compte de toutes sortes, médiatrice pour les abandonnés, elle est placée sous la protection de cet autre "prophète sans visage», appartenant tout à la fois à la mythologie gréco-romaine et arabe, Hermès, l'ami des marginaux, déchiffreur des signes, Mercure vif-argent et encore Idris bâtisseur des cités. Comment dès lors, porteuse de toute cette histoire, n'aurait-elle pas refait le monde? «Fugitive» (p. 219) à son tour, elle est de retour dans son désert, portant son enfant, conçu dans une grotte, avec un ancien esclave, sous les tonnerres de Zeus. Elle, ainsi qu'Agar seule face à la maternité du monde, une Agar qui aurait renversé les rapports de classes ou encore Marie vue par les musulmans, comme un signe de Dieu. Par ailleurs, Lalla répète une situation similaire à celle vécue par sa mère pour la mettre au monde comme le rapporte par le récit de Amma : «elle était debout contre l'arbre, avec les bras accrochés aux branches» (p. 88), récit qui se continue ainsi : «et c'est dans le manteau bleu de ta mère que tu as dormi.» (p. 89) Accrochée à

I8. É. Brehier, Histoire de la philosophie [1931, 1938], PUF, 1980. 
son tour au vieux figuier par une ceinture, elle vit les mots mêmes qui désignent la souffrance de l'accouchement : «Tenir la corde» ou «Bonne cordée» sont en arabe les souhaits que l'on adresse à toute femme au moment du travail. Lalla à son tour revit la même expérience : «Son manteau marron est étendu par terre... Elle accroche la ceinture à la pièce maitresse branche du figuier» (p. 42I), puis c'est le cri de la vie qui éclate, la toilette dans l'eau de mer, et le nom donné à l'enfant selon la lignée maternelle : le mythe a tout renversé. Hawa, fille de Hawa enveloppée dans son manteau aura son récit. 